\title{
Development of a Wideband Current Amplifier dedicated to Fission Chamber Measurement
}

\author{
Danilo Bisiach $^{3}$, Loïc Barbot ${ }^{1}$, Grégoire De Izarra ${ }^{1}$, Christophe Destouches ${ }^{2}$, Manuel Cargnelutti ${ }^{3}$ and Sebastjan \\ Zorzut $^{3}$ \\ ${ }^{1}$ CEA, DES, IRESNE, DER, SPESI, Instrumentation Sensors and Dosimetry Laboratory, Cadarache, F-13108 \\ Saint Paul-lez-Durance, France \\ ${ }^{2}$ CEA, DES, IRESNE, DER, Experimental Physics, Safety experiment and Instrumentation Section, Cadarache, \\ F-13108 Saint Paul-lez-Durance, France \\ ${ }^{3}$ Instrumentation Technologies, d.o.o., Velika pot 22 - 5250 Solkan - Slovenija, Slovenia \\ danilo.bisiach@i-tech.si
}

\begin{abstract}
Fission chambers are widely used in nuclear reactors, either occasionally to probe the neutron flux maps in nuclear power plants or as part of the nuclear instrumentation used in research reactors to characterize neutron flux levels in irradiation locations.

Wide dynamic range acquisition systems are very limited in the nuclear instrumentation market. Thus, since 2012, CEA is developing the MONACO system, which allows measurement in pulse, fluctuation, and current mode running simultaneously on the same channel.

The simultaneous use of three measurement modes places high requirements on the amplification stage. Indeed, the current mode exploits a bandwidth between 0 and a few $\mathrm{kHz}$, while the fluctuation mode is based on the frequency range of $100 \mathrm{kHz}-1 \mathrm{MHz}$. Finally, for the pulse mode, it is necessary to identify current pulses of a few uA in amplitude and with a duration of about ten nanoseconds, which implies a bandwidth that extends up to 10 or 20 MHz.
\end{abstract}

In the frame of the industrialization of the MONACO system, a new preamplifier was designed to meet those requirements. With a first prototype, LDCI and I-TECH performed very promising bench tests in October 2020. Tests with synthetic signals corresponding to pulse and fluctuation mode showed very good performance of the electronics. The positive and negative current pulses are correctly amplified and the measurement noise was estimated at $0.1 \mu$ App thanks to proper electronic shielding. The DC measurement is also satisfactory in the tested range with an accuracy of less than $\mathrm{nA}$ and a bandwidth of $1 \mathrm{kHz}$. Preamplifier qualifications are planned within realistic experiments using a fission chamber placed in a neutron field.

Keywords - Miniature Fission Chamber, Neutron Flux Measurement, Transimpedance amplifier, Low Current Measurement

\section{INTRODUCTION}

$\mathrm{T}^{\mathrm{s}}$ HE MONACO system [1][2] (Multichannel Online Neutron Acquisition in Campbell mOde) was developed at CEA during the past 10 years until the TRL7 level and demonstrated its efficiency as a functional prototype for Fission Chamber (FC) signal acquisition. The main feature of the system is to make possible to acquire simultaneously three different FC working mode: pulse, Campbelling and, current mode. Within the commissioning of the new Jules Horowitz nuclear reactor, this system will be integrated into the experimental part of the data acquisition system. The industrialization of the MONACO system is a result of a mutual efforts between CEA who developed the prototype and Instrumentation Technologies. The industrialized version of the MONACO system will be available on the market from 2023 and will be supported for at least 10 years aftermarket.

One of the key parts of the MONACO system is the frontend amplifier originally developed by CEA and based on analog components. Instrumentation Technologies redesigned the preamplifier to meet CEA performance requirements using new discrete and high-performance components.

The entire redevelopment process was divided into different phases, including a CEA specific PO for the first phase and a bilateral collaboration agreement for the remaining phases, to lower the risk connected to every step of industrialization:

- Phase 1: Redevelopment of the integrated preamplifier unit. This phase will consist of the redevelopment of the preamplifier frontend and of the modules that are critical for the MONACO system performance: the HV module, and the current measurement unit. This phase was successfully concluded and will be described in the next paragraphs of this paper.

- Phase 2: Integration of a 4-channel system based on the hardware prototype developed in phase 1 . This phase will expand the 1-channel integrated frontend to a fully functional 4-channel system with the integration of a digitizer unit aimed to collect and process the digitalized data. The hardware developed within this phase will consist in the finalized version of the MONACO system First testing at a research reactor facility to confirm the hardware performance are planned in August 2021.

- Phase 3: Development of the Digital Signal Processing (DSP) interface on the digitizer module integrated into the 4-channel system.

- Phase 4: Development of the user Graphical User Interface (GUI) software and final testing of the device.

This paper will cover the first phase of the process where a functional pre-industrialization 1-channel version of the preamplifier was developed and tested. 


\section{INTEGRATED PREAMPLIFIER REQUIREMENTS}

The MONACO system developed by CEA[MOL] is based on different building blocks that fulfill the final user application requirements. Each of the 4 channels is composed of a processing chain with the following blocks:

- Preamplifier frontend: aimed to collect the signal from the FC.

- High voltage source (independent for all the 4 channels) that polarizes the FC.

- Current measurement unit (independent for all the 4 channels) that measures the current provided to the MFC.

- Digital acquisition board and FPGA processing.

- Host PC with GUI.

The block system is well described in Fig.1.

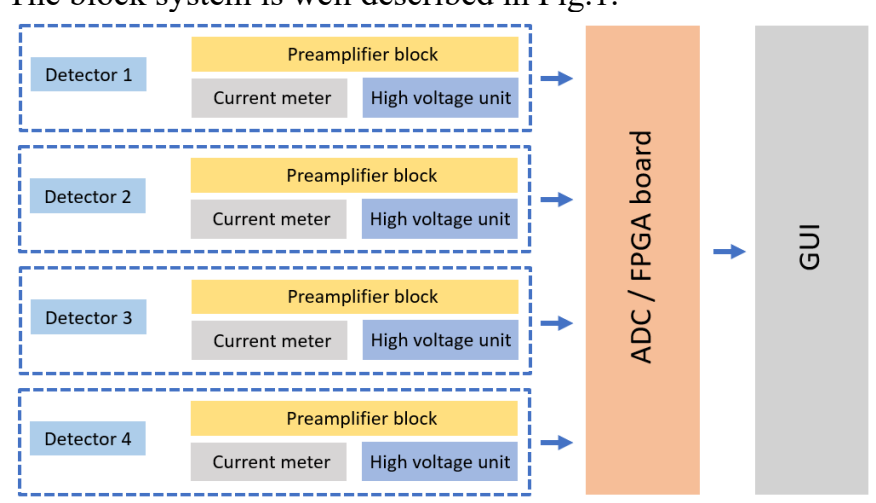

Fig. 1. The 4 channel MONACO system block schematics

In the following paragraphs, we will refer to the "preamplifier frontend" as the frontend unit which is converting and amplifying the signals from the FC, and to "integrated preamplifier" as the unit that was developed by Instrumentation Technologies that integrates the preamplifier frontend, the HV module, and the current meter.

Within Phase 1, the goal is to provide a 1-channel prototype that can replicate the preamplifier frontend, high voltage source, and current meter unit integrated into a standalone system that can lead to the same or better results than the existing MONACO prototype developed by CEA.

The preamplifier development began with the review of the specifics of the actual MONACO system. As described in Fig. 1 the unit is composed of three separated modules, the preamplifier frontend, an HV unit, and a current measurement unit connected with wires in a common enclosure. All these blocks should work as an integrated subsystem, so the first choice was to integrate all the units on a common PCB to simplify the communication between the units and to improve EMC (noise immunity).

The preamplifier frontend converts the current signal retrieved from the $\mathrm{FC}$ detector into a voltage signal and amplifies it with some specific performance parameters in terms of gain and noise. The performance reference parameters were taken from the measurements of the existing CEA preamplifier.

The HV unit needs to deliver the polarization voltage to the detector with a $\pm 1 \mathrm{kV} \pm 1 \%$ accuracy and a maximum of 1 $\mathrm{mA}$ output for each channel.

The current measurement unit provides an operative range from $10 \mathrm{nA}$ to $1 \mathrm{~mA}$ with $1 \mathrm{nA}$ of resolution at lower currents and integrates a digital sampling system with a sampling rate of $1 \mathrm{kS} / \mathrm{s}$.

\section{INTEGRATED PREAMPLIFIER DEVELOPMENT}

In this paragraph, we will review the development of the preamplifier frontend, $\mathrm{HV}$, and current meter unit. As reported before the starting point was the review of the performances of the existing CEA preamplifier. This unit seemed to be the most critical for the system performance since it is responsible for the HV decoupling, noise/disturbances immunity of the system, and overall performance in terms of bandwidth.

The block schematics of the integrated preamplifier is described in Fig.2:

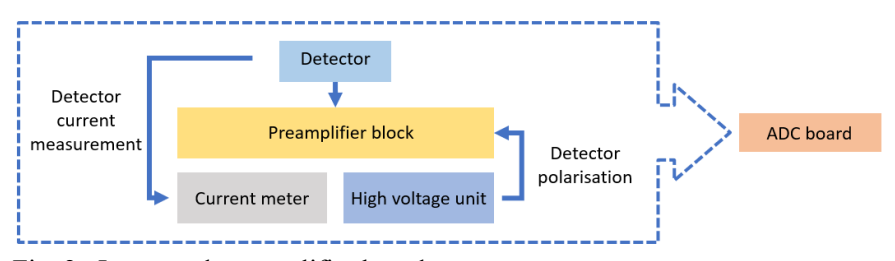

Fig. 2. Integrated preamplifier board concept

As mentioned before the choice to integrate all the modules on a common PCB will allow easier integration and interface debugging during the system development. Having a common interface for unit power supply and communication will also make easier the extension from the 1-channel to the 4-channel system.

\section{A. Testing of the original CEA frontend preamplifier}

The CEA preamplifier, delivered as a sealed box, consists of 1-channel electronics capable to collect the signal from the FC with given performance specifications and some additional features such as cable capacity immunity and the possibility to deliver also polarization voltage using an external high voltage source up to $\pm 1 \mathrm{kV}$. The CEA preamplifier parameters were measured at Instrumentation Technologies and are listed in Table I, these values will be the reference values for the I-Tech preamplifier development:

TABLE I

CEA PREAMPLIFIER SPECIFICATIONS

\begin{tabular}{ll}
\hline \hline \multicolumn{1}{c}{ Feature } & Specifications \\
\hline Bandwidth & $10 \mathrm{kHz}-18 \mathrm{MHz}$ \\
Transimpedance gain & 17500 \\
Output noise & $0.2 \mu \mathrm{A}$ peak to peak \\
Cable capacitance immunity & $\sim 50 \mathrm{nF}$ \\
& $20 \mathrm{uA}$ \\
Current limit & \\
\hline \hline
\end{tabular}

The CEA preamplifier interfaces are composed of an $\mathrm{HN}$ input connector, two lemo output connectors, fine and coarse trimmers for gain adjustment and calibration, DC supply input. The electronics are packed and shielded in a high immunity enclosure.

The noise measured on the CEA preamplifier without any termination on the $\mathrm{HN}$ input was $40 \mathrm{mVpp}$. By attaching a 23 
$\mathrm{m}$ long shielded high-immunity cable the total noise figure increased to $50 \mathrm{mVpp}$, demonstrating the strong cable capacity immunity of the preamplifier.

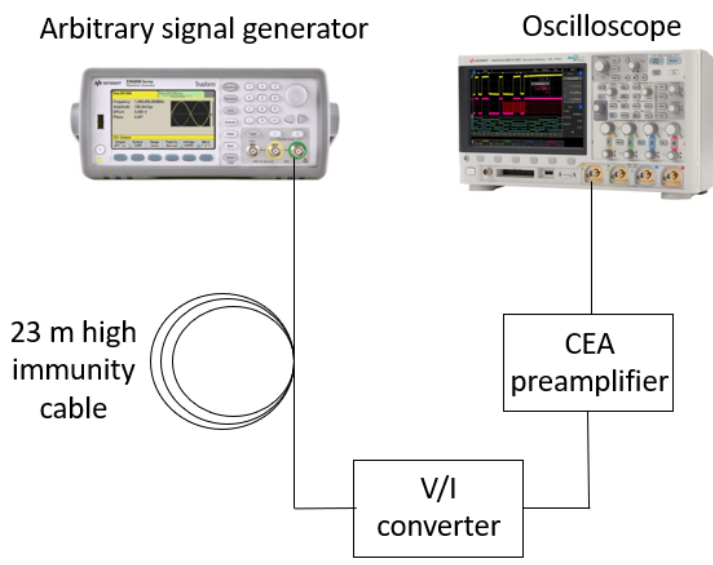

Fig. 3. The measurement setup of the CEA frontend preamplifier consisted of a Keysigth/Agilent arbitrary signal generator in combination with a Keysigth InfiniiVision DSOX3104T $1 \mathrm{GHz}, 5 \mathrm{GSa} / \mathrm{s}$ oscilloscope

The next step was the evaluation of the effects of pulse signal reflection in the cable. Negative and positive pulses with different widths were provided to the preamplifier, from 20 $\mathrm{ns}$ to $200 \mathrm{~ns}$ width. The reflection time was influencing all the pulses depending on the pulse width. An example of a $20 \mathrm{~ns}$ pulse response is reported in Fig. 1:

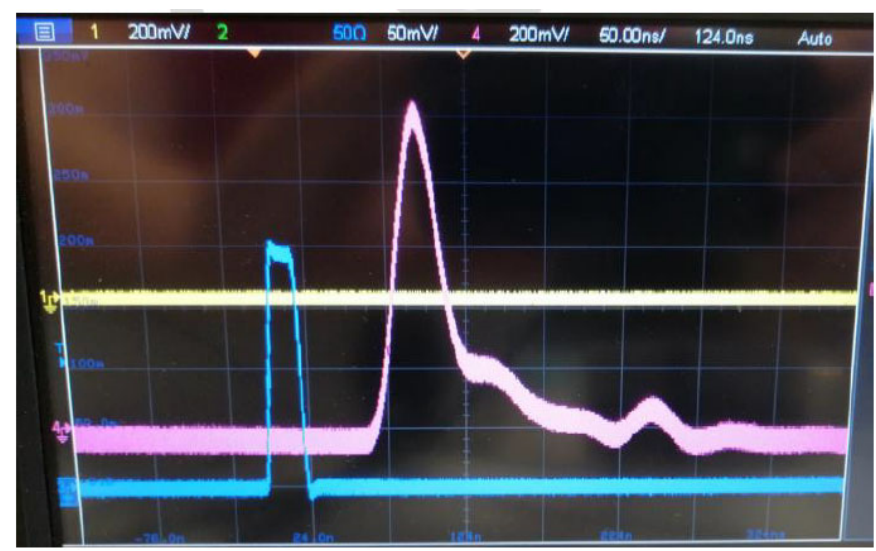

Fig. 4. A 20 ns positive pulse (blue) was delivered to the $\mathrm{I} / \mathrm{V}$ converter through a $23 \mathrm{~m}$ high immunity long cable. The amplifier response (pink) presented a $10 \mathrm{~ns}$ rise time with a reflection occurring after the pulse bounced through twice the cable length.

The preamplifier S parameters were tested with an Agilent E5052B Signal Source Analyzer. The total bandpass was confirmed to be around $20 \mathrm{MHz}$ as reported in the S21 parameter plot in Fig. 5.

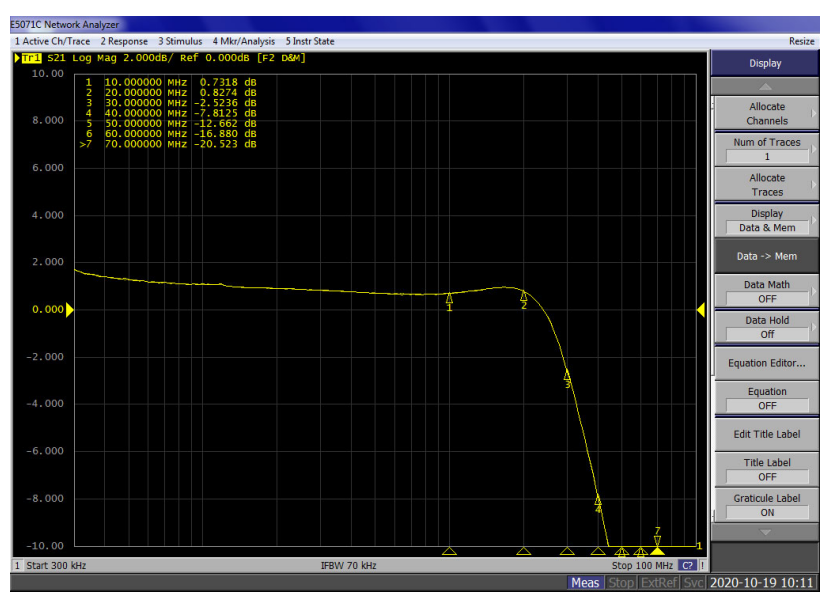

Fig. 5. CEA preamplifier S21 parameter during bandwidth evaluation Marker 2 is positioned at $20 \mathrm{MHz}$.

\section{B. I-Tech integrated preamplifier development}

Based on the requirements listed in paragraph $\mathrm{A}$, the Instrumentation Technologies preamplifier was redesigned by using discrete components. As described before, since this module needs to integrate all the key components of the final MONACO system (preamplifier, HV source, and current meter), special attention was given to noise immunity and DC-DC converter shielding. All these blocks will find their place on a dual-side PCB with a strong amplifier stage shielding as reported in Fig.6.

The preamplifier input is retrieved by a $\mathrm{HN}$ connector and a decoupling condensators allows the further stages to get only the AC component without the HV bias that is provided to the detector.

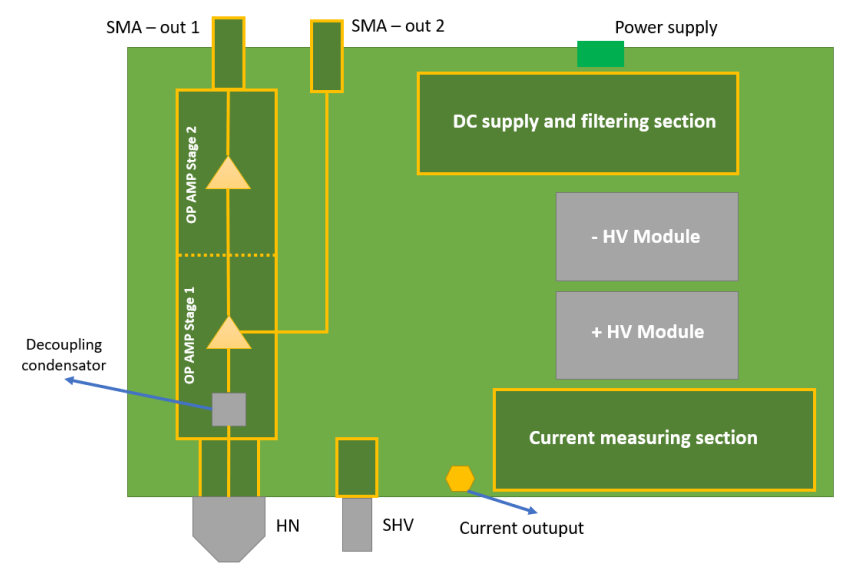

Fig. 6. Integrated preamplifier sections: the preamplifier chain is divided into two subchains and can provide the $\mathrm{HV}$ polarisation and the current measurement.

To investigate different amplification levels and to allow a quicker circuit debugging, the preamplifier frontend was split into two different gain outputs that were compared within the same pulse signal, both outputs are 50 Ohms matched. Different configurations between stages 1 and 2 lead to different pulse responses and noise figures. The $\mathrm{HN}$ connector shielding plays an important role in the prevention of external interferences. Additional connectors allow to retrieve the $\mathrm{HV}$ value (SHV connector) and the current that is provided from the $\mathrm{HV}$ modules.

\section{I-Tech HV unit}

The HV unit was designed using negative and positive HV modules that were combined in a common circuit separated by a relay. It allows the user to set the high voltage value 
within the $+1 \mathrm{kV}$ to $-1 \mathrm{kV}$ range. Strong filtering on the connector output was needed to prevent the ripple noise and to level the voltage level within the specifications. The addition of output leveling capacitors also leads to review the safety certification needs due to the amount of charge that can be released by the system.

\section{I-Tech current meter unit}

The current meter was designed using a shunt resistor system and by amplifying the voltage drop on this resistor with an operational amplifier chain. The main difficulties during the development of the unit came from the parasitic currents and the shielding of the unit. The measurement of few $n A$ resolution within several hundred volts was possible by adding additional shielding to the unit and by isolating it within the PCB on a floating reference.

\section{INTEGRATED SYSTEM PROTOTYPE}

All the units listed in the previous paragraphs (preamplifier frontend, HV module, current measurement module) were integrated on a single PCB board that also includes the power supply unit and part of the control logic. This proof of concept was also lately applied to the effective MONACO prototype by extending the setup to a 4-channel board and adding the $\mathrm{ADC}$ acquisition and control system.
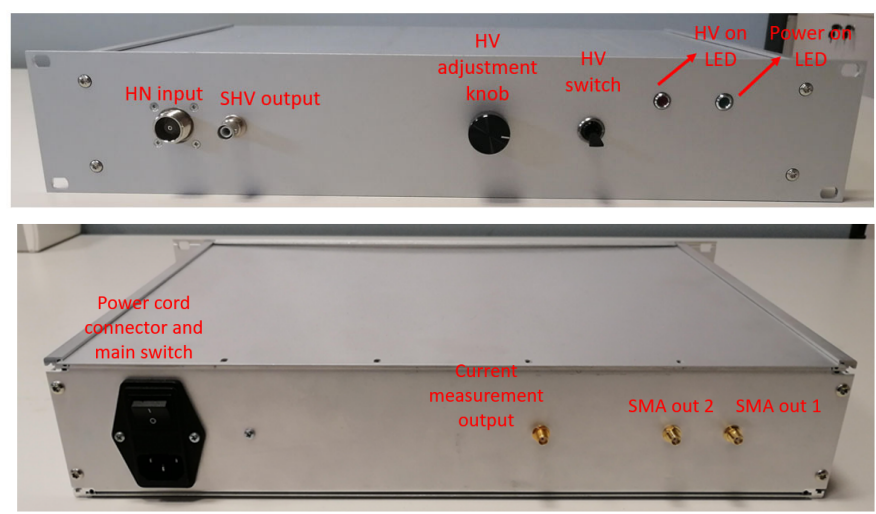

Fig. 7. Preamplifier integrated sections: the preamplifier chain is divided into two subchains and can provide the HV polarization and the current measurement.

The hardware enclosure allows the testing of the integrated preamplifier by shielding the inner components which are very sensitive to external perturbations. The power supply was also integrated in the final design. The input signal from the detector is delivered to the HN connector and the HV can be adjusted with an external knob. The outputs, as mentioned before, allow the user to evaluate the signal from the preamplifier stages (1 and 2) and from the current measurement section.

\section{Performance Measurement}

\section{A. Preamplifier frontend evaluation}

The system performance was evaluated by simulating the pulse train using an Agilent 33250A/ $80 \mathrm{MHz}$ Arbitrary Waveform Generator and a Tektronix AWG5012 arbitrary 1 $\mathrm{GHz}$ signal generator. Within this setup it was possible to assess the bandwidth and the rise time of the preamplifier frontend and to confirm the absence of unexpected behaviors (e.g., noise, spikes, ringing...).

The pulse measurements were performed both for positive and negative pulses and the performance was comparable to the existing CEA preamplifier with an increased bandwidth so with a lower rising time of the measured pulses.

The preamplifier frontend bandwidth was later lowered to prevent the increase of the noise figure and of the cable ringing effect that was noticed lately during the preamplifier frontend comparisons.

Within the finalized prototype, the base noise level of the preamplifier was measured to be $40 \mathrm{mVpp}$ but with some spikes coming from the DC/DC converters on the prototype board at approximately $100 \mathrm{kHz}$. With the use of ad-hoc low drop-out integrated into the DC supply module the total noise figure improved.

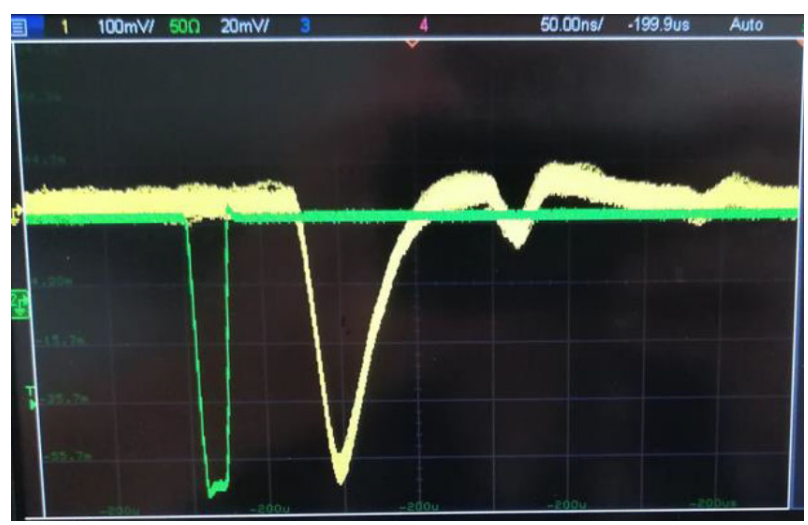

Fig. 8. Instrumentation Technologies frontend preamplifier negative pulse response. The oscillations after the pulse are due to the cable.

The S21 parameters showed a high increase in the amplifier bandpass:

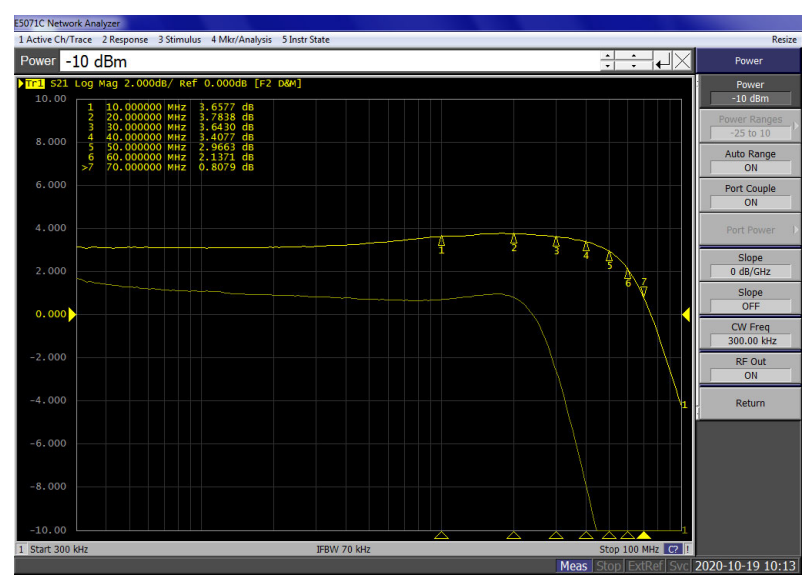

Fig. 9. Instrumentation Technologies preamplifier frontend S21 parameter (upper curve) vs CEA preamplifier frontend (lower curve)

By injecting white noise in the preamplifier frontend and recording the spectral response on an oscilloscope the bandwidth was confirmed. The addition of the $23 \mathrm{~m}$ length high immunity mineral cable did not influence significatively the noise figure even if some reflections were noticeable. In any case, the cable reflections are not estimated as a showstopper for the implementation of the DSP algorithm that will be running on MONACO. With this test, the result confirmed the performance of the Instrumentation Technologies amplifier to be similar to the original CEA preamplifier frontend. 


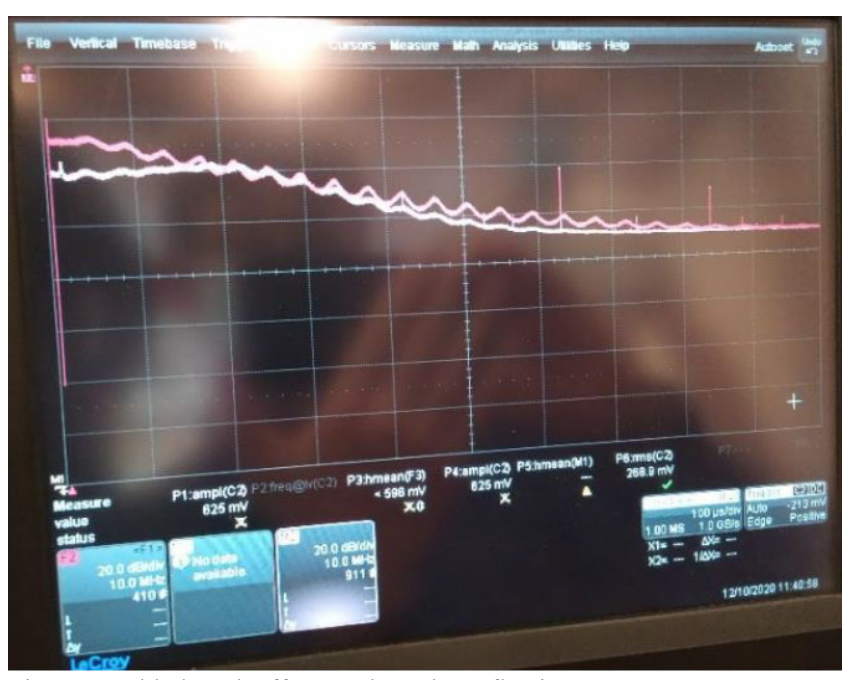

Fig. 10. Cable length effect on the pulse reflections.

\section{B. High voltage module performance}

The high voltage module was tested for high voltage stability and the presence of ripples. The overall performance was linked to the output capacitors' ability to smooth the incoming noise from the DC supply modules. The measurements showed a maximum of $1 \mathrm{kV}$ with a ripple below $60 \mathrm{mV}$.

\section{Current meter module performance}

The overall performance of the unit was measured to be in the range of $4 \mathrm{nA}$ and will be lowered in the next designs.

The operative range from 0 to $1 \mathrm{kV}$ was measured by comparing the current value measured by the current meter unit and the Keithley 6517B Electrometer/Resistance meter. The results are reported in Fig. 11 where strong linearity is seen between the two systems. A $100 \mathrm{MOhm}$ resistor was connected to the HV output and the relative current was measured. The data relate very well in the entire span from 0 to $-350 \mathrm{~V}$ with a final difference at the highest voltage and current value due to the prototype amplifier saturation (green box).

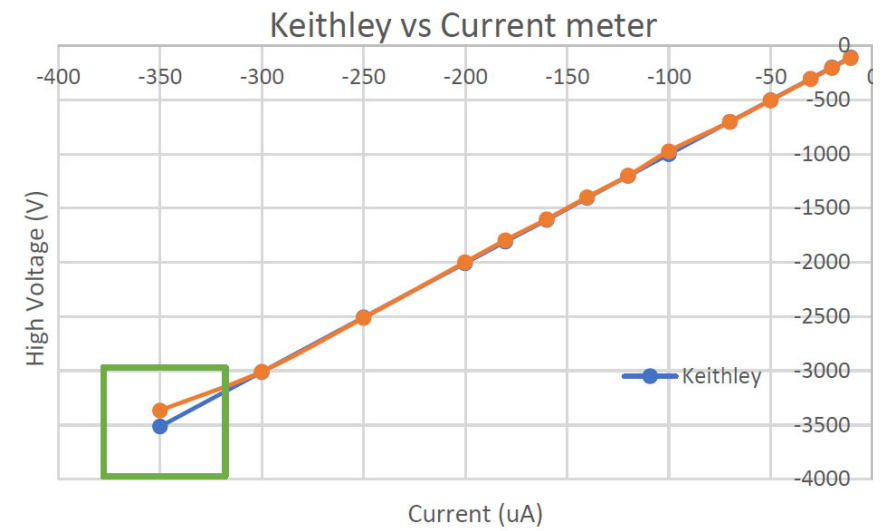

Fig. 11. I-Tech vs Keithley 6517 current measurement performance. A shunt resistor was polarized with the $\mathrm{HV}$ output and the relative current was measured from 0 to $-350 \mathrm{~V}$.

The bandpass of the current meter was measured using a sinusoidal signal from the Keithley current source, with a sweep from $50 \mathrm{~Hz}$ with measured amplitude $35.1 \mathrm{mV}$ to $1 \mathrm{kHz}$ with measured amplitude $15.2 \mathrm{mV}$. This confirmed the bandpass point of $-3 \mathrm{~dB}$ of the current meter to be $1 \mathrm{kHz}$.

\section{CONCLUSIONS}

The development of the integrated preamplifier prototype was the first milestone and the proof of capability for the redesign of the MONACO system's main building blocks. The results reported in the measurements allowed Instrumentation Technologies to evaluate all the critical points of the preamplifier, HV module, and current meter unit. Some of the main difficulties were due to the preamplifier design (cable capacitance...) and logistics due to the actual Covid19 pandemic (prototype testing, electronic components delivery).

The integrated preamplifier was also tested at the CEA facility with a neutron source, the results will be reported in the next publications.

The next steps of industrialization are already in progress with the foreseen delivery of the 4-channel integrated version of MONACO as a hardware prototype in July 2021. This unit will allow the confirmation of the 4-channel hardware architecture and the start of the CE certification procedure.

The MONACO industrialized version is planned to be available on the market in early 2023.

\section{REFERENCES}

[1] L. Barbot, D. Fourmentel, G. De Izarra, C. Destouches, J-F. Villard, Y. Moline and H. Hamrita, "MONACO v2: Multipurpose and Integrated Data Acquisition System for On-line Neutron and Gamma Measurements", ANIMMA 2019, June 17-21, 2019, Portoroz, Slovenia.

[2] Y. Moline, A. Dabat-Blondeau, H. Hamrita, S. Klasa, G. Corre, J-M. Bourbotte, K. Boudergui, L. Barbot, C. Destouches, "MONACO: A Case Study of a New System on Chip and Stream Based Architecture for Nuclear instrumentation", ANIMMA 2019, June 17-21, 2019, Portoroz, Slovenia. 